\title{
¿POR QUÉ HABLAR DE INTERCULTURALIDAD EN RELACIÓN CON LA EDUCACIÓN Y LAS CIENCIAS SOCIALES?: PROYECTO DE Posdoctorado EN EduCACIÓn, CiEnCIAS Sociales E INTERCULTURALIDAD EN LA UNIVERSIDAD SANTO TOMÁS DE BOGOTÁ
}

Millones de personas en todo el mundo sobreviven sometidas a la pobreza y la exclusión social
y no es probable que en los próximos años estas condiciones vayan a desaparecer. Esta grave
situación atañe a toda la humanidad que no puede ni debe cerrar los ojos ante ella. Mientras
persista, todos estamos un poco empobrecidos y somos en cierto modo excluidos (Estivill, 2003).

José Arlés Gómez Arévalo

En la época contemporánea, América Latina y el Caribe, regiones cada día más complejas y paradójicas, cuentan con nuevos entornos internacionales integrados por algunos

1 Posdoctor en Ciencia-Narrativa, doctor en Teología, magíster en Filosofía Latinoamericana, especialista en Educación Sexual, especialista en Educación y Filosofía Colombiana, licenciado en Teología. Actualmente director del grupo Ciencia-Espiritualidad y autor de varios artículos y libros sobre educación, epistemologías emergentes, espiritualidad y teología. Correo: angel777abc@hotmail.com 
aspectos que sitúan a la interculturalidad como un imperativo y signo esperanzador de su presencia en el concierto mundial; sin embargo, según las cifras proporcionadas por las entidades oficiales (Unesco, OIT, Cepal, OEA, entre otras), la brecha entre ricos y pobres sigue creciendo, las naciones se ven abocadas a nuevas formas de xenofobia, exclusión, marginación y violencia, factores que constituyen bombas de tiempo que deben preocupar a sus naciones y a sus gobiernos, de cara al presente y al futuro inmediato de sus pueblos.

Si bien en estas regiones existe una mayor conciencia y sensibilización hacia la pluralidad cultural, frente al encuentro con el otro y con lo otro-incluso frente al fenómeno de la globalización hay una mayor visibilidad de las desigualdades, exclusiones, formas de violencia social y política, así como de género y de creencias religiosas, entre otras-, aún existen algunas situaciones que no han supuesto cambio sino, por el contrario, la continuidad del statu quo en términos de desigualdad, separación y falta de alteridadsolidaridad, así como de diálogo y tolerancia. Se trata de un continente y de una región del mundo cada vez más signada por los alarmantes abismos entre sus pueblos, condenados al olvido y la marginación, en un modelo neoliberal que impone cada día con más fuerza sus reglas de juego económicas y políticas y en donde los grandes perdedores son la inmensa mayoría de hombres, mujeres, niños y ancianos que habitan las periferias y los conglomerados humanos de las grandes ciudades de América Latina.

Frente a la falta de proyectos fuertes de integración y de intercambio sociocultural y económico, el actual marco histórico presenta el proceso de «globalización» como fenómeno representante de una política y estrategia que se expande mundialmente como «la opción» civilizadora, imponiéndose así como el proyecto para la humanidad, en un intento homogenizador contradictorio a una propuesta intercultural.

En este marco referencial, complejo y dispar, antitético y antinómico, se propone hablar de «interculturalidad», categoría que, se debe reconocer, no posee una definición unívoca; el debate conceptual sobre la misma está lejos de terminar, en la actualidad existe un verdadero mosaico de perspectivas y aproximaciones, propias de un término eminentemente polisémico. En palabras de Dietz (2001), el mismo concepto de estudios interculturales ha sido propuesto para distinguir «un campo emergente de preocupaciones transdisciplinarias en torno a los contactos y las relaciones que a nivel individual como colectivo se articulan en contextos de diversidad y heterogeneidad cultural» (p. 23). En el fondo, el autor pone sobre la mesa la urgencia de reflexionar sobre los trabajos en el área de las ciencias sociohumanísticas, las cuales han sido por tradición las abanderadas de la 
crítica y el análisis de nuevas perspectivas sobre las problemáticas del hombre, la cultura y la sociedad actual.

Cabe destacar también la posición de Vallescar (2010), quien asevera que la interculturalidad se levanta abiertamente frente a un proyecto de la modernidad marcado por el progreso sin límite, por el triunfo del más apto y fuerte que no requiere de nadie. He aquí una razón fundamental para la interculturalidad: hacer frente a una modernidad uniformizante, aportando a la construcción de un futuro diverso y plural para todos.

Hablar de interculturalidad es, pues, sacar a la luz muchos de los conflictos que existen en nuestras sociedades, es aceptar que hay diferentes culturas y que no todas tienen el mismo reconocimiento y poder (Torres, 2008). Este reconocimiento de la diversidad obliga a cuestionar las desigualdades y todo tipo de consecuencias que conllevan (pobreza, discriminación, racismo, xenofobia, etc.). Encontramos aquí otra poderosa razón para la interculturalidad: contribuir al cuestionamiento de las situaciones estructurales y de las condiciones que permiten que haya dominación de unas culturas sobre otras, de determinados colectivos humanos sobre otros, etiquetados como diferentes e inferiores.

Después de este breve recorrido por algunas de las posturas sobre la interculturalidad, cabe preguntarse ¿a qué se hace referencia cuando se menciona la categoría interculturalidad? El término interculturalidad alude al encuentro entre (inter) culturas, lo cual nos lleva en primer lugar a una necesaria aproximación (porque asumimos la dificultad de definirla) a lo que entendemos por cultura. La cultura está presente en el conjunto de formas o modos particulares de expresar la vivencia del mundo y de la vida, en la cerámica, el teatro, la danza, la música, pero no solo como producción tangible sino como el conjunto de formas y modos adquiridos de concebir el mundo, de pensar, de hablar, de expresarse, de percibir, de comportarse, de organizarse socialmente, de comunicarse, de sentir y valorarse a uno mismo en cuanto individuo y en cuanto grupo (Heise, Tubino y Ardito, 1992). Desde esta perspectiva todos los seres humanos hacemos cultura y todos somos portadores de cultura.

\section{Interculturalidad y su relación con la educación y las ciencias sociales}

La interculturalidad es una dimensión que no se limita solo al campo de la educación, sino que se encuentra presente en las interacciones humanas en general como alternativa frente al autoritarismo, el dogmatismo y el etnocentrismo. Sin embargo, la búsqueda 
de sociedades más democráticas y plurales supone procesos sociales y educativos que afirmen y proporcionen la experiencia de vivir en democracia y la experiencia del respeto a la diversidad. Como educadores tenemos una responsabilidad ineludible.

Cabe mencionar aquí el debate que la Organización de Naciones Unidas para la Educación, la Ciencia y la Cultura, Unesco, presenta en su texto de Directrices sobre la Educación Intercultural. Dicho debate teórico sobre educación y multiculturalismo, así como sobre los principales aspectos e interrelaciones, da cuenta, aunque de manera muy breve y sistémica, de las complejidades que implica la aproximación a esta racionalidad teorética. La discusión teórica presente en este discurso pone sobre la mesa un asunto latente en función de estas cuestiones: la vinculación del multiculturalismo con problemáticas de las culturas minoritarias dentro de la cuales es posible identificar a los pueblos autóctonos, las minorías territoriales y no territoriales y los inmigrantes. Asimismo, allí se puntualiza que la interculturalidad es un concepto mucho más dinámico y más amplio; siguiendo con esta línea de reflexión, es menester rescatar el enfoque de educación intercultural planteado por la Unesco, donde el diálogo, el conocimiento mutuo y el respeto son los ejes para la convivencia evolutiva y sostenible en las sociedades interculturales.

De la misma manera, algunos autores, entre ellos López (2008), aseguran que la interculturalidad en la educación aparece estrechamente ligada al nuevo espíritu de equidad y calidad que inspiran las actuales propuestas educativas, lo que contribuye a la superación de la visión igualitarista que predominó en el escenario social latinoamericano desde la llegada de las primeras oleadas liberales al continente. López (2008) nos dice que la interculturalidad en la educación supone un doble camino: hacia adentro y hacia fuera, y que una de las direcciones necesarias a las que debe dirigirse un proyecto educativo intercultural, particularmente cuando está dirigido a pueblos que han sido objeto de opresión cultural y lingüística (como los nuestros), es precisamente hacia las raíces de la propia cultura y de la propia visión del mundo, para estructurar o recomponer un universo coherente sobre el cual se pueda luego cimentar, desde una mejor posición, el diálogo e intercambio con elementos culturales que, si bien ajenos, son necesarios tanto para sobrevivir en el mundo de hoy como para alcanzar mejores niveles de vida, aprovechando aquellos avances y desarrollos científico - tecnológicos que se consideren necesarios.

La educación intercultural debe entenderse inmersa en un proceso pedagógico que involucra a varios sistemas culturales. Nace del derecho individual y colectivo de los pueblos ancestrales y/o migrantes que comprende no solo el derecho a gozar de la educación como todos los ciudadanos/as, sino también del derecho a mantener y cultivar sus propias tradiciones, 
su propia cultura, sus propios valores. Asimismo, comprende la necesidad de desarrollar competencias interculturales que permitan a cualquier ciudadano de cualquier lugar del país pertenecer o no a la cultura hegemónica y convivir democráticamente con los otros.

Una manera de pensar una propuesta de educación intercultural es abordándola desde los diferentes ámbitos de acción: hacia uno mismo y sus pertenencias (identidad), hacia el otro (convivencia), o hacia el mundo referencial (conocimiento). A continuación se explicitan dichos ámbitos.

\section{Identidad}

Es el lugar de las «pertenencias» del individuo. El objetivo en ese campo es lograr que los educandos asuman con libertad sus pertenencias y tradiciones, que las valoren y sean capaces de establecer entre ellas un vínculo fecundo e innovador.

\section{Convivencia}

Es el ámbito de la interacción con el otro, del «vivir juntos», de los derechos de la exigibilidad; es el ámbito en el que se construye una «ciudadanía intercultural». El desafío de una educación intercultural en este campo es el de lograr que los educandos generen una ética de la reciprocidad en los diversos circuitos en los que interactúan.

\section{Conocimiento}

Es el ámbito del conocimiento del mundo referencial. Hay diferentes formas de percibir el mundo, de conceptualizarlo y representarlo. El desafío de una educación intercultural en este campo es el de romper la lógica de una sola fuente y forma de conocimiento.

A nivel de las ciencias sociales, la interculturalidad supone la búsqueda de relaciones positivas entre personas de diferentes culturas; ello implica el encuentro de un yo (nosotros) con un otro (los otros) y para que este encuentro se lleve a cabo en el marco de relaciones positivas se ha tenido que trabajar en lo que Xavier Albo (2003), llama «los dos polos necesarios», la identidad (desde el yo) y el reconocimiento del otro (alteridad). 
La propia identidad se define por los compromisos e identificaciones que proporcionan el marco u horizonte dentro del cual yo intento determinar lo que es bueno, lo que es valioso, lo que se debe hacer, lo que apruebo o a lo que me opongo. En otras palabras, es el horizonte dentro del cual puedo adoptar una postura (Taylor, 1994). Lo que entendemos por «identidad», se trata de «quién» somos y «de dónde venimos», constituye el trasfondo en el que nuestros gustos y deseos, opiniones y aspiraciones, cobran sentido.

La revalorización cultural es un aspecto fundamental de la interculturalidad, pues solamente en la medida en que las culturas tradicionales refuercen su autoestima será posible una relación de horizontalidad democrática y no de verticalidad dominante con la cultura de las sociedades contemporáneas.

Al afianzamiento de la propia identidad le sigue el camino hacia fuera, es decir, la actitud de apertura, respeto y acogida hacia los otros que son distintos por venir de culturas distintas, quizás incluso desconocidas. No se acepta a alguien simplemente por ser «mejor», ni se lo rechaza por ser «peor», sino que, por principio, se le acoge con apertura a pesar de ser distinto y quizás desconocido (Albó, 2003).

Asimismo, en el campo del conocimiento se da un conflicto entre el conocimiento consagrado en el ámbito educativo y los saberes locales, excluidos y menospreciados. Un enfoque intercultural se abrirá a indagar sobre los saberes, valores y prácticas locales, a fin de incorporarlos en el trabajo cotidiano; esa apertura hacia lo local también debe incluir el conocimiento de la historia. La incorporación de los saberes, valores y prácticas locales no se limita a la identificación de los saberes previos de los alumnos, sino que merece un espacio de trabajo definido en el cual se explore su significado y sus aportes; ese espacio educativo deberá ser debidamente contextualizado recuperando escenarios y formas de aprendizaje locales (Tubino, 2011).

En esta misma línea, la interculturalidad en la educación pondría de relieve la pertenencia cultural de los sujetos y su relevancia social, es decir, el patrimonio cultural de los diferentes, poniendo en juego la idea de García Canclini (2004) de que «el umbral de la ciudadanía» se conquista no solo obteniendo el respeto a las diferencias sino contando con los «mínimos competitivos en relación con cada uno de los "recursos capacitantes" para participar de la sociedad: trabajo, salud, poder de compra» (p. 82).

El recorrido hacia la educación intercultural quizás sea largo, pero nuestras expectativas están puestas en los pequeños cambios que día a día los profesores se arriesguen a realizar en sus aulas, reconociendo la igualdad del otro como un principio fundador; también, en que más temprano que tarde los actores de poder hagan suya la idea de que la «la educación 
intercultural propone la igualdad de derechos y deberes en la teoría y en la práctica y prepara el terreno para una convivencia pacífica en la diferencia» (Sáez, 2008, p. 122).

Cabe mencionar aquí el trabajo investigativo del equipo encargado del posdoctorado en Educación, Ciencias Sociales e Interculturalidad, que se direcciona bajo la guía de cuatro componentes esenciales que se explican a continuación:

\section{Componente educativo-pedagógico}

Este componente pone en evidencia la necesidad de un entendimiento de la educación como objeto de estudio de la pedagogía. El proceso de enseñanza-aprendizaje es una especie de núcleo de la educación, no obstante, no es en esencia un proceso educativo. La educación es un hecho social, político, económico, ético o moral, filosófico, axiológico, psicobiológico, administrativo, jurídico, ambiental, histórico-cultural o intercultural, entre otros, pero en todos ellos predomina un carácter y sello de clase social. El proceso de enseñanzaaprendizaje es la acción de enseñar y aprender al margen del carácter y sello de clase social, se trata de un proceso instruccional o tecnológico cuya relación con la educación depende principalmente del educador más que del educando.

Es importante entender cómo se le ha definido a la educación. Según José F. Lora (2006), «la educación es la formación integral de la personalidad humana: moral, artística, intelectual, física, etc., con la finalidad de adaptar a los educandos a una determinada sociedad» (p. 40). Por otra parte, Aníbal Ponce (1970) sostiene que «la educación en las sociedades de clases antagónicas ha servido y sirve -en el sentido estricto de la expresióna los intereses económicos, políticos, ideológicos, etc., de las clases sojuzgadoras» (p. 32). Otra definición, idealista por supuesto, que cabe resaltar es la de Lorenzo Luzuriaga (1990), quien afirma que «la educación es, por lo pronto, un hacer, una actividad que tiene por fin formar, dirigir o desarrollar la vida humana para que ésta llegue a su plenitud» (p. 126).

En la misma línea, cabe anotar que la educación es un objeto de estudio de una ciencia cardinal llamada pedagogía, así como de sus ciencias complementarias entre las que se encuentran la filosofía de la educación; la sociología de la educación; la psicología educativa; la historia de la educación; la didáctica o tecnología educativa; la antropología de la educación; la administración educativa; la ética y axiología, en función a las clases sociales populares; la biología, con su teoría de la biología del aprendizaje; la neurolingüística, entre otros campos del conocimiento. 
Las pedagogías emergentes necesariamente contarán con una comunidad conformada por profesionales de la Educación, por profesionales de otras carreras y por estudiantes de Educación que reconocen la importancia de la educación intercultural para la edificación de la identidad de los pueblos latinoamericanos, desde los paradigmas del mundo globalizado. Asimismo, partirán de una concepción científica de la realidad - de la biodiversidad en la naturaleza y de la sociedad multicultural, multiétnica y multilingüe-, desde una nueva conciencia social intercultural, con elevada vocación de servicio y aptitud para la producción de bienes materiales e intelectuales, en el amplio contexto de Latinoamérica y del Caribe.

\section{Componente sociedad}

La sociedad que ampara, soporta o estimula a la pedagogía emergente está constituida por toda la población de América Latina multicultural, multiétnica y multilingüe, inmersa en un escenario paradigmático, entre un desarrollo socioeconómico marcado por el modelo neoliberal y la pobreza y miseria de grandes masas de su población; estos fenómenos son producto de los propios líderes políticos en arreglo con sus precursores ideológicos de corte neocolonialista, transnacional y capitalista.

Las grandes masas de población latinoamericana necesitan con urgencia una nueva educación, ya que la que recibieron (y reciben aún) carece de discursos y tendencias democráticas, dialogantes y abiertas al pluralismo multi e intercultural. Las consecuencias de esta educación tradicional están a la vista de todos: fenómenos como la deserción, la frustración, la inhibición, el bajo rendimiento, la confusión e incompetencia de los egresados, el bajo nivel de acceso a la educación superior y la falta de oportunidades. El fracaso de muchos sistemas educativos no es accidente social, es la tragedia social, porque la educación es parte del fundamento de la naturaleza humana. La realidad descrita obliga a repensar la educación; queda una vía aún inexplorada: la educación intercultural.

\section{Componente filosófico-ontológico}

Este componente contribuye a analizar el ser social en transformación permanente, así como los elementos del ser del educando, del principio epistemológico de que el mundo puede conocerse objetivamente (al menos parcial y gradualmente), y del ethos de la libre 
búsqueda de la verdad, profundidad, comprensión y sistema «más que, digamos, el ethos de la fe religiosa o el de la búsqueda de información por la información, utilidad, beneficio, poder, consenso o el bien» (Bunge, 1999).

Desde la ontología, el educador en el contexto de la pedagogía emergente es aquel que cuenta con el requisito fundamental e inexcusable de poseer una concepción científica del mundo, una conciencia social, una vocación de servicio y una habilidad para producir bienes materiales e intelectuales. El educando, por su parte, es el sujeto principal en la educación y quien participa activamente influenciado por un determinado sistema educativo.

Esta pedagogía, entonces, implica un ethos que se interesa por la búsqueda de la verdad, profundidad, comprensión y sistematización de las cuestiones que atañen dicha verdad. Se trata de una pedagogía emergente para la educación intercultural que tiende hacia la unidad y el diálogo, desde la diversidad, para la edificación de la identidad de los pueblos latinoamericanos, en el contexto histórico de más de veinticinco siglos transcurridos; en un lento proceso de decantación de la cultura occidental que ha permitido la formación de estructuras básicas y petrificadas, las cuales sirven de columna vertebral a las distintas teorías, tecnologías y prácticas sociales, económicas y educativas, en la época contemporánea.

\section{Componente cultural}

En este componente se propone analizar la categoría interculturalidad, aquella que se ha convertido en una herramienta crítica para analizar los conflictos que existen en nuestras sociedades, aquella que propone el tema del diálogo entre las diferentes culturas y hace énfasis en que no todas tienen el mismo reconocimiento y poder (Torres, 2008). El reconocimiento de la diversidad obliga a cuestionar las desigualdades y todo tipo de consecuencias que conllevan (pobreza, discriminación, racismo, xenofobia, etc.). Desde esta perspectiva, la interculturalidad contribuye a cuestionar las situaciones estructurales y las condiciones que permiten que haya dominación de unas culturas sobre otras, etiquetadas como diferentes e inferiores.

Aquí es importante precisar el término interculturalidad, no sin antes establecer la diferencia entre pluralismo cultural, multiculturalismo e interculturalidad. Siguiendo a C. Giménez Romero (2004), hay que distinguir entre el plano fáctico (de los hechos) y el plano normativo o de las propuestas sociopolíticas y éticas. En el plano fáctico, Giménez sitúa 
la multiculturalidad (diversidad cultural, lingüística, religiosa, etc.) y la interculturalidad (relaciones interétnicas, interlingüísticas, interreligiosas, etc.). En el plano normativo incluye el multiculturalismo (reconocimiento de la diferencia) y el interculturalismo (convivencia en la diversidad). Todos estos fenómenos son modalidades del pluralismo cultural.

\title{
II. Propuesta de seminarios posdoctorales
}

Como un intento de responder a las inquietudes ya planteadas, desde una mirada de formación posgradual y en términos académicos, se proponen los siguientes seminarios de profundización en el posdoctorado en Educación, Ciencias Sociales e Interculturalidad.

\section{Seminario $n^{\circ}{ }^{\circ}$ : Interculturalidad y su relación con pedagogías emergentes en Latinoamérica y el Caribe}

\begin{abstract}
Mientras que los latinoamericanos no nos eduquemos para una convivencia intercultural, no podremos entendernos, ni integrarnos. Pese a que se habla mucho del tema de la interculturalidad y la diversidad, se ha avanzado muy poco y es un grupo minoritario quien lo pone en práctica y lo impulsa Hipólito Percy, 2013.
\end{abstract}

\section{Planteamiento general del seminario}

Este seminario se propone abordar los temas relacionados con la problemática de la interculturalidad, desde su relación con las pedagogías emergentes a lo largo y ancho de Latinoamérica y del Caribe.

Así mismo, aborda la existencia de todo un movimiento pedagógico enfocado en no solo responder al reto que significa la realidad de una sociedad multicultural y étnicamente plural, sino también en implicarse en el proyecto de sociedad futura, ya emergente, y abordar su significado desde las implicaciones de esta realidad plural y compleja, caracterizada por el intercambio y la movilidad de las personas, de los sistemas de valores y de los modelos culturales y sociales. 
Por último, con base en la problemática de la convivencia en un lugar de pluralidad cultural y étnica, se plantean las posibilidades de desarrollo de una auténtica pedagogía intercultural e inclusiva que posibilite prácticas, investigaciones y reflexiones, desde el campo educativo y de las ciencias sociales, que ofrezcan principios básicos y propuestas de acción concretas desde la compleja realidad de Latinoamérica y del Caribe.

Principios y propuestas de acción desde la pedagogía intercultural

Vázquez (1994) establece como presupuestos pedagógicos que constituyen la base para regir la acción educativa intercultural, los siguientes principios:

- Antropológicos: identidad, diálogo y diversidad.

- Epistemológicos: existencia de algunos valores universales comunes a las diversas culturas.

- Praxiológicos y tecnológicos: programas educativos viables y eficaces.

\section{Preguntas problema que direccionan el seminario}

Según los planteamientos anteriores, se formula una pregunta que constituye el derrotero de búsqueda a nivel de la educación, de la pedagogía y de las ciencias sociales, con relación al problema de la interculturalidad y las pedagogías emergentes:

¿Es posible elaborar una propuesta educativa y social sobre el problema de la interculturalidad, que construya nuevos modelos teóricos que establezcan un corpus científico a nivel pedagógico y que brinde respuestas a dicha problemática?

¿Desde qué presupuestos las pedagogías emergentes, de acuerdo a los estándares que caracterizan a las ciencias sociohumanísticas, pueden repercutir en una educación intercultural que constituya la fuerza orientadora del sentido de las prácticas sociales y educativas en Latinoamérica y el Caribe? 


\section{Objetivos:}

1. Intercambiar experiencias desde el campo de las ciencias sociales y desde las prácticas educativas que tienden al fortalecimiento, promoción y desarrollo de pedagogías que fomentan el diálogo intercultural en América Latina y el Caribe.

2. Describir conceptualmente las propuestas de las pedagogías emergentes y su aporte a la educación intercultural, para formentar el respeto y valoración de la diversidad multicultural, multilingüe y multiétnica, y así contribuir a la edificación de la identidad de los grupos sociales en América Latina y el Caribe.

3. Fomentar el diálogo intercultural desde las prácticas y diversos enfoques pedagógicos emergentes que combaten la exclusión, el racismo, la segregación y discriminación, desde una nueva perspectiva de la diversidad sociocultural de las comunidades latinoamericanas y caribeñas.

\section{Líneas temáticas}

- Los estudios interculturales como campo emergente de la inter y transdisciplinariedad de las ciencias humanas y sociales en la época actual.

- Creación de espacios interculturales en contextos educativos emergentes.

- El diálogo intercultural desde las prácticas educativas y desde diversos enfoques pedagógicos emergentes.

- Tensiones y dilemas propios de una sociedad globalizada, tecnocrática e intercultural.

- Problemas teóricos y prácticos de una educación intercultural en el contexto latinoamericano y caribeño.

- La educación intercultural como respuesta ante las crisis de las sociedades contemporáneas. 


\title{
Seminario $n .^{\circ}$ 2: problemáticas curriculares en contextos de interculturalidad en Latinoamérica y el Caribe
}

\begin{abstract}
Un currículo intercultural debe apoyarse en las miradas cotidianas de lo cultural, porque desde ella se construye y se reconstruye la realidad dinámica de la realidad latinoamericana y caribeña, porque a través de ella se saca a la luz las problemáticas de la sociedad y del mundo, estructuramos nuevas racionalidades para su comprensión y se ofrecen nuevas estrategias pedagógicas y didácticas para la comprensión del mundo de la vida a partir de la educación.
\end{abstract} Eduardo Restrepo, 2004.

\section{Objetivos}

Revisar las propuestas y modelos curriculares que tienen en cuenta el fomento de la diversidad étnica, lingüística y cultural, así como la producción regional de nuevas miradas educativas y de las prácticas pedagógicas sustentadas en el respeto a las diferencias étnicas, religiosas y socioculturales.

Crear un ambiente de diálogo y debate para que los participantes puedan comunicar, desde sus experiencias educativas sobre los procesos de enseñanza, educación, aprendizaje y formación, y desde los muchos enfoques curriculares latinoamericanos y caribeños.

Abrir un espacio para el análisis de los diversos elementos que entran en el currículo intercultural, desde una visión holística e integradora de los diversos procesos socioculturales de Latinoamérica y del Caribe que tienden a la inclusión y el respeto por la diversidad.

\section{Planteamiento general del seminario}

\section{La diversidad cultural en los procesos de construcción curricular}

En este seminario se trata de considerar al currículo educativo como la herramienta clave para orientar a docentes y estudiantes en la construcción de sentido de identidad cultural en Latinoamérica y el Caribe, desde sus procesos de formación, a través de las máximas expresiones artísticas, sociales y educativas de nuestras regiones. 
Asimismo, el seminario reflexiona sobre los nuevos espacios interculturales por medio de los cuales los actores educativos pueden comunicar sus apreciaciones, con la mejor intención de conocer y orientar los procesos de enseñanza, educación, aprendizaje y formación, procesos con singularidades diversas que tienen lugar mediante currículos latinoamericanos y caribeños, que los adaptan según las necesidades particulares.

Otra reflexión de este seminario es sobre la correspondencia entre currículo educativo y la pertinencia cultural. Para ello se debe cuestionar si se está asegurando, mediante los proyectos pedagógicos, la generación de espacios de reflexión intercultural de tal manera que tengan relevancia en la región y en el continente; asimismo, se deben plantear las puestas en escena de todos los fenómenos socioculturales y políticos que afectan a nuestra sociedad contemporánea.

Por último, es importante considerar los currículos con pertinencia intercultural, los mismos que vienen a configurar un nuevo lenguaje que permite promocionar la identidad cultural local, regional, nacional y latinoamericana. Esta promoción contribuye al proceso de reconstrucción del tejido social desde la escuela, la familia y el estado. De esta manera, se establece una nueva relación de situación y de postura entre el currículo, la cultura para ser leída y la educación vista como la máxima expresión de la sociedad, puesto que la sociedad se ve representada y movilizada desde lo educativo, esto es, da a conocer sus preocupaciones culturales para que el currículo constituya una relación indisoluble con la cultura, dinamice las prácticas pedagógicas y promocione el encuentro con lo formativo, en cuanto que contribuye a construir cotidianamente los imaginarios culturales desde el aprendizaje intercultural.

\section{Pregunta problema que direcciona el seminario:}

Para comprender la importancia de los currículos en contextos interculturales, se hace necesario responder a las preguntas: ¿cómo el currículo se convierte en una estrategia formativa en contextos de diálogo intercultural? y ¿qué estrategias se pueden implementar para promover la interculturalidad en los currículos actuales en Latinoamérica y el Caribe?

Esto, desde luego, nos lleva a «mirar» más allá de unos contenidos temáticos o de las lógicas del proceso de enseñanza y aprendizaje, nos lleva a establecer niveles de comprensión de nuestra identidad cultural para que sea la manera de ver y la manera de divulgar nuestros valores. Esta mirada desde currículos interculturales se debe trabajar desde los proyectos pedagógicos como una mirada que toque la cotidianidad cultural desde la escuela, mediante las informaciones que los actores sociales nos ofrezcan. 


\section{Líneas temáticas}

- Miradas semiológicas a la construcción curricular desde los discursos interculturales, con referencia a lo educativo-pedagógico.

- Construcción de proyectos pedagógicos que aborden la pertinencia intercultural desde el currículo: qué es y cómo debe ser un currículo intercultural en América Latina y el Caribe.

- La construcción de currículos interculturales, con pertinencia, como respuesta a los procesos identitarios de lo local, lo regional y lo internacional.

- La pertinencia del currículo intercultural como significación sociocultural a través de los proyectos educativos y pedagógicos.

- La educación como juego dialéctico que se expresa en un currículo pertinente, que llega desde el quehacer cotidiano de la educación: relación entre lo curricular y la realidad cultural.

- Relación entre la sociedad, la escuela y el contexto intercultural en la elaboración del currículo.

Seminario n. $^{\circ} 3$ Gestión del conocimiento: repensar la educación y las ciencias sociales en clave de interculturalidad en América Latina y el Caribe

Una pedagogía del encuentro intercultural exige un cambio de nuestra lingüística. Se entiende por cultura al conjunto de comportamientos, suposiciones, formas culturales, prejuicios, creencias, actitudes, estereotipos, opiniones sobre el lenguaje en general y sobre las lenguas en particular. Gualdieri y Vásquez, 2011.

\section{Objetivos}

1. Generar un espacio de diálogo para analizar y debatir las relaciones entre la educación y las ciencias sociales, en el concierto de la globalización del conocimiento y su relación con el fenómeno de la interculturalidad en América Latina y el Caribe. 
2. Pensar en la posibilidad y necesidad de estrategias asociativas entre la educación y las ciencias sociales para dar respuesta a las problemáticas inherentes al interculturalismo, en y desde América Latina y el Caribe, en diálogo con la comunidad científica internacional.

3. Realizar algunas propuestas a nivel de educación y ciencias sociales en lo referente al diálogo intercultural desde la complejidad de la globalización del conocimiento y de la ciencia en la época contemporánea.

\section{Planteamiento general del seminario}

Este seminario abre el espacio para reflexionar sobre el problema de la globalización del conocimiento desde una nueva visión del papel de la educación y de las ciencias sociales en los procesos de transformación de América Latina y del Caribe. Durante los últimos años, a partir del establecimiento de procesos de transformaciones sociales en varios países de la región, en los cuales las políticas de lucha contra la pobreza y por el desarrollo social han adquirido un lugar prioritario en el conjunto de las políticas públicas, se ha planteado un escenario más favorable para la articulación de un diálogo más constructivo entre educación, ciencias sociales e interculturalidad.

En las décadas anteriores, ciertos consensos internacionales generados en los países latinoamericanos y caribeños de mayor desarrollo -basados esencialmente en los criterios de la globalización, la competitividad y la rentabilidad a todo costo, así como en un pragmatismo apoyado en la desregulación de los mercados, la deconstrucción del estado y la subordinación de los intereses nacionales a una lógica global-, cerraron el paso a una participación más dinámica, constructiva e influyente de la educación y de las ciencias sociales en la región, así como a una participación en los procesos políticos de la mayoría de sus países.

No se puede afirmar que la situación actual es menos compleja que la anterior, más bien al contrario, pero lo que sí se puede afirmar es que la superación de la ortodoxia, dominante por años, ha cedido el paso a un nuevo periodo de búsqueda de alternativas desde la región, que da lugar a mejores condiciones para el diálogo entre nuevas perspectivas educativas, las ciencias sociales y las políticas públicas al respecto. El desafío es enorme, este va desde la necesidad de construir nuevas teorías explicativas de los principales problemas educativos y sociales de la región latinoamericana y caribeña, así como de su 
lugar en el mundo, hasta la evaluación de las políticas en curso y la contribución al diseño de mejores alternativas. Se trata de un trabajo de carácter crítico, como no podría dejar de serlo en tanto que científico, pero a la vez comprometido con un curso de acción a favor de los mejores intereses de la educación y de la sociedad.

Cabe mencionar aquí la visión de Schmelkes (2002) sobre el tema intercultural con relación a la educación y a las ciencias sociales; él sostiene que la interculturalidad implica la toma de una postura política que debe promover el cambio de las relaciones de asimetría que caracterizan la sociedad homogeneizadora y globalizada. En este caso, la interculturalidad implica una relación respetuosa entre culturas, donde se pone énfasis en la superación de tres tipos de asimetrías: cultural, social y educativa. Esta última asimetría podría ser la clave para la construcción de un continente y una región intercultural, ya que no puede ocurrir un cambio de fondo sin una actividad educativa fuerte y decisiva que permee las estructuras sociales y las transforme desde su raíz.

La Comisión Económica para América Latina y el Caribe (Cepal) y la Organización de las Naciones Unidas para la Educación, la Ciencia y la Cultura (Unesco) advertían ya hace una década que:

Al convertirse el conocimiento en el elemento central del nuevo paradigma productivo, la transformación educativa pasa a ser un factor fundamental para desarrollar la capacidad de innovación y la creatividad, a la vez que la integración y la solidaridad, aspectos clave tanto para el ejercicio de la moderna ciudadanía como para alcanzar altos niveles de competitividad (Cepal/Unesco, 1992, p. 119).

Preguntas problema que direccionan el seminario:

¿De qué manera se pueden articular la educación y las ciencias sociales desde el diálogo intercultural, en la sociedad de la globalización del conocimiento, para dar respuesta a las principales problemáticas socioculturales y educativas en América Latina y el Caribe? ¿Qué estrategias se pueden implementar desde la educación para dar respuesta al malestar cultural de las sociedades latinoamericanas y caribeñas, teniendo en cuenta los aportes de la ciencia y el conocimiento en la época de la globalización y planetización? 


\section{Líneas temáticas}

1. Complejidad y paradojas del conocimiento y de la ciencia en la era de la globalización y de la comunicación.

2. Diálogo entre educación y ciencias sociales desde los retos de la interculturalidad en América Latina y el Caribe.

3. Claves para la construcción de propuestas educativas en los nuevos escenarios sociales del continente latinoamericano y caribeño, a partir del diálogo con el conocimiento y las ciencias actuales.

4. Análisis de las condiciones para el diálogo entre nuevas perspectivas educativas, las ciencias sociales y las políticas públicas en Latinoamérica y el Caribe

\section{Seminario n. $^{\circ}$ : Investigación en ciencias sociales y mediáticas en la sociedad del conocimiento en Latinoamérica y el Caribe}

\section{Planteamiento general del seminario}

Este seminario se propone realizar una reflexión sobre el fenómeno de la interculturalidad y su relación con las ciencias sociales y mediáticas, así como con las ciencias de la educación, que han debido convivir con los cambios sociales generados por la extrema pobreza, la inequidad de género, la violencia, la erosión de las culturas campesinas, los problemas relativos a la justicia y la mercantilización e industrialización de la comunicación social.

En estos marcos de crisis y de exclusión, el seminario profundiza sobre la investigación en ciencias sociales y mediáticas, las cuales han debido detenerse a analizar la movilización de grandes masas de desplazados, de indígenas y campesinos, de comunidades marginadas a lo largo y ancho de la geografía latinoamericana y caribeña. Por esta vía comienzan a darse los primeros síntomas de un colapso en los objetivos de cambio cultural e intragrupal y de una cierta impotencia para lograr el rediseño de las prácticas pedagógicas que declaran la legitimación del discurso de la interculturalidad educativa. Sin embargo, también se fortalece una poderosa y emergente corriente de etnización o profundización de políticas específicas de identidades sociales, en donde se destacan las acciones afirmativas en pro de la defensa de los indígenas, los inmigrantes, los niños y las mujeres, principales poblaciones excluidas de los procesos de cambio cultural y educativo en América Latina y el Caribe. 
Por otra parte, el seminario contribuye a la reflexión sobre los acelerados cambios que impone la sociedad de la información en el ámbito productivo y comunicacional, los cuales obligan a una rápida y ágil adaptación por parte de los nuevos medios para la transmisión de conocimientos, la comunicación a distancia y el uso de información. En el área educativa, más que contenidos curriculares, se requiere generar una disposición general al cambio en las modalidades de aprendizaje, comunicación y producción.

En este marco, es urgente mejorar la calidad y pertinencia del sistema educacional a fin de que este cumpla una función estratégica en el tránsito de las sociedades nacionales hacia un orden global, competitivo y altamente interconectado, centrado en el paradigma de la sociedad del conocimiento. Es necesario, además, armonizar los progresos educativos con otro pilar de la sociedad de la información, a saber, el acceso al intercambio comunicacional por medios audiovisuales e interactivos, donde no solo se juega la competitividad sino también la identidad cultural y, cada vez más, la participación ciudadana; de allí la importancia crucial de las mediaciones entre educación, industria cultural y las nuevas Tecnologías de la Información y de la Comunicación (TIC). Educar con estas tecnologías implica educar para imprimirle al uso de las TIC sentidos que compatibilicen las nuevas formas de producir y trabajar con los actuales estilos de ejercer derechos, afirmar culturas, informarse, comunicarse a distancia y formar parte de redes investigativas a nivel de la educación y de las ciencias sociales en América Latina y el Caribe.

\section{Objetivos}

1. Profundizar en el estado de la investigación en ciencias sociales y mediáticas en la sociedad del conocimiento en Latinoamérica y el Caribe para contribuir con nuevas propuestas investigativas que fomenten el diálogo entre la educación y las ciencias sociales desde la perspectiva de la interculturalidad.

2. Analizar las corrientes de etnización o profundización de políticas específicas de identidades sociales, en donde se destacan las acciones afirmativas en pro de la defensa de los indígenas, los inmigrantes, los niños y las mujeres, principales poblaciones excluidas de los procesos de cambio cultural y educativo en América Latina y el Caribe.

3. Reflexionar sobre los acelerados cambios que impone la sociedad de la información en el ámbito productivo y comunicacional, que obligan a los nuevos medios 
masivos a una rápida y ágil adaptación, en relación con la cultura y la educación, para la transmisión de conocimientos, la comunicación a distancia y el uso de información.

4. Generar una disposición general al cambio en las modalidades de aprendizaje, comunicación y producción, desde el diálogo con la interculturalidad latinoamericana y caribeña.

\section{Líneas temáticas}

1. Educación, comunicación y cultura en la sociedad de la información: una perspectiva latinoamericana y caribeña.

2. La transmisión de conocimientos, la comunicación en el ámbito educativo y el uso de la información en el concierto de la interculturalidad en Latinoamérica y del Caribe.

3. Las mediaciones entre educación, industria cultural y las nuevas Tecnologías de la Información y de la Comunicación (TIC).

4. El estado de la investigación en ciencias sociales y mediáticas en la sociedad del conocimiento en Latinoamérica y el Caribe.

\section{Conclusión}

La propuesta de Posdoctorado en Educación, Ciencias Sociales e Interculturalidad constituye un punto de referencia académico e investigativo -en una época marcada por la enorme brecha entre ricos y pobres, por la xenofobia, la exclusión, la marginación y la violencia-, especialmente en la reflexión sobre la importancia de adquirir una mayor conciencia y sensibilidad frente a la pluralidad cultural, así como frente a la alteridad, al encuentro con la tolerancia y el pluralismo sociocultural, incluso frente al fenómeno de la globalización y mundialización de la sociedad contemporánea, en el que hacen más visibles las desigualdades, las exclusiones, las formas de violencia social y política, así como de género y religión, entre otras.

Así pues, se trata de generar un espacio de reflexión para discutir el papel de la educación y de las ciencias sociales en la realidad de una región del mundo cada vez más 
signada por la falta de proyectos fuertes de integración y de intercambio sociocultural y económico, así como por los alarmantes abismos entre sus pueblos, condenados al olvido y a la marginación, en un modelo neoliberal que impone cada día con más fuerza sus reglas de juego económicas y políticas y en donde tanto la educación como las ciencias sociales tienen mucho que aportar desde estatutos epistemológicos y su reflexión teórica-práctica.

\section{Referencias}

Albó, X. (2003). Iguales aunque diferentes. En Módulo Interculturalidad, Especialidad en Educación Intercultural. Perú: Editorial PUCP.

Bunge, M. (1999). Fundamentos de biofilosofia. México, D.F.: Siglo XXI Editores.

Dietz, G. (2001). Del multiculturalismo a la interculturalidad: Un movimiento social entre discurso disidente y praxis institucional. En de Prado, J. (Ed.), Diversidad cultural, identidad y ciudadanía (pp. 17-71). Córdoba: Instituto de Estudios Transnacionales.

García Canclini, N. (2004), Diferentes, Desiguales y Desconectados, Mapas de la Interculturalidad. Barcelona, España: Editorial Gedisa.

Giménez Romero, C. (2004). Pluralismo, Multiculturalismo e Interculturalidad. En Educación y Futuro, (8). Madrid, España: Ediciones Don Bosco.

Heise, M. Tubino, F. y Ardito, W. (1992). Interculturalidad: un desafío. Lima, Perú: Editorial CAAP.

López, L. (2008). Algunas claves para entender la interculturalidad en la educación (PROEIB). Perú: Editorial Asociación de Publicaciones Educativas.

Lora Cam, J. (2006). Mitologías universales y latinoamericanas. $1^{\mathrm{a}}$ ed. Lima. Editores - impresores Juan Gutemberg.

Luzurriga, L. (1990). Historia de la Educación y la Pedagogía. Buenos Aires, Argentina: Editorial Losada.

Percy, H. (2013). Pensamientos educativos. Lima, Perú: Editorial PUCP.

Ponce, A. (1970). Educación y lucha de clases. Buenos Aires, Argentina: Ediciones Imago Mundi.

Restrepo, E. (2004). Políticas curriculares en tiempos de multiculturalismo: proyectos educativos de/ para afrodescendientes en Colombia. En Currículo sem Fronteiras, 12(1), pp. 157-173.

Sáez Alonso, R. (2008). La educación intercultural en el ámbito de la Educación para el Desarrollo Humano Sostenible. En Revista Complutense de Educación, 12(2), pp. 713-737.

Schmelkes, S. (2002). La interculturalidad en educación básica. En Encuentro Internacional de Educación Preescolar: Curriculum y Competencias. México, D.F.: Editorial Santillana. 
Taylor, Ch. (1994). La ética de la autenticidad, Barcelona, España: Editorial Paidos.

Torres, C. A. (2008). Después de la tormenta neoliberal: la política educativa latinoamericana entre la crítica y la utopía. En Revista Iberoamericana de Educación, (48), pp. 207-229. .

Tubino, F. (2011). Interculturalidad para todos: ¿un slogan más?. Lima, Perú: Editorial PUCP.

Vallescar D. (2010). Interculturalidad, creación de un concepto y desarrollo de una actitud. Lima, Perú: Ediciones Lux.

Vásquez, J. C (1994). La interculturalidad: Un debate necesario en el Perú III. En Revista Construyendo Nuestra Interculturalidad. Recuperado de:http://interculturalidad.org/numeroo4/o1o1pdv.htm 